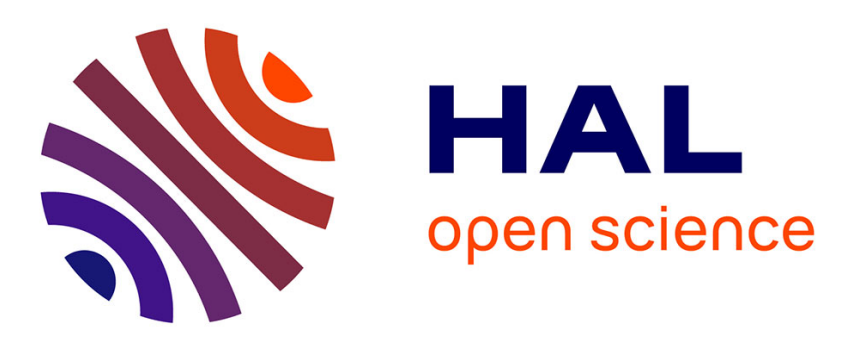

\title{
Symbolic Monte Carlo method applied to the identification of radiative properties of a heterogeneous material
}

Yassine Maanane, Maxime Roger, Agnès Delmas, Mathieu Galtier, Frédéric

André

\section{To cite this version:}

Yassine Maanane, Maxime Roger, Agnès Delmas, Mathieu Galtier, Frédéric André. Symbolic Monte Carlo method applied to the identification of radiative properties of a heterogeneous material. Journal of Quantitative Spectroscopy and Radiative Transfer, 2020, 249, pp.107019. 10.1016/j.jqsrt.2020.107019 . hal-02613374

\section{HAL Id: hal-02613374 \\ https://hal.science/hal-02613374}

Submitted on 20 May 2020

HAL is a multi-disciplinary open access archive for the deposit and dissemination of scientific research documents, whether they are published or not. The documents may come from teaching and research institutions in France or abroad, or from public or private research centers.
L'archive ouverte pluridisciplinaire HAL, est destinée au dépôt et à la diffusion de documents scientifiques de niveau recherche, publiés ou non, émanant des établissements d'enseignement et de recherche français ou étrangers, des laboratoires publics ou privés. 


\title{
Symbolic Monte Carlo method applied to the identification of radiative properties of a heterogeneous material.
}

\author{
Yassine Maanane $^{\mathrm{a}}$, Maxime Roger ${ }^{\mathrm{a}, *}$, Agnès Delmas $^{\mathrm{a}}$, Mathieu Galtier ${ }^{\mathrm{a}}$, \\ Frédéric Andréa \\ ${ }^{a}$ Univ Lyon, CNRS, INSA-Lyon, Université Claude Bernard Lyon 1, CETHIL UMR5008, \\ F-69621 Villeurbanne, France
}

\begin{abstract}
A Symbolic Monte Carlo method (SMC) is applied to the identification of radiative properties of a heterogeneous semitransparent insulating material from measurements of directional-hemispherical transmittance and reflectance at room temperature. The polynomials obtained with SMC allow the development of a complete inverse analysis which determines if the inverse problem solution exists, is unique and stable. Moreover, the numerical efficiency of the absorption and scattering coefficients identification is improved since the radiative transfer equation is only solved once in the overall inverse iterative procedure.

Keywords: Radiative Transfer, Symbolic Monte Carlo (SMC), Polynomials, Radiative properties, Identification, Insulating materials
\end{abstract}

\section{Introduction}

Inverse problems in radiative transfer concern many applications including the characterization of complex media (porous or fibrous $[1,2]$, combustion diagnosis [3], medical imaging $[4,5]$, etc.). Identification methods combine spectroscopic measurements with simulations based on radiative models, such as the radiative transfer equation (RTE), in order to infer parameters such as

\footnotetext{
* Corresponding author

Email address: maxime.roger@insa-lyon.fr (Maxime Roger)
}

Preprint submitted to Journal of Quantitative Spectroscopy \& Radiative TransferApril 6, 2020 
7 radiative properties, temperature or species concentrations. For instance, spectral measurements of transmitted and reflected fluxes in different configurations (bidirectional, directional-hemispherical, hemispherical-hemispherical ...) can be used to identify the effective scattering and absorption properties of various media $[6,7,8]$. In these approaches, inversion is performed by using an iterative procedure, where at each step, direct computations and measurements are compared.

In the frame of inverse radiative transfer problems, Symbolic Monte Carlo methods (SMC) turns out to be a powerful and efficient tool for inverse analysis and to improve the efficiency of identification method. The principle of SMC consists in retaining some parameters as symbols in a Monte Carlo simulation, in order to express the observable as a simple and unbiased function of those symbolic parameters [9]. The observable is therefore estimated all over the parameter space, which is valuable for inversion analysis purpose. SMC (initially labeled inverse Monte Carlo method) was introduced by Dunn [10] and investigated for inverse problems in radiative transfer in [10, 11]. Dunn [10] identified the scattering albedo in an inhomogeneous medium assuming isotropic scattering. The approach was extended by Subramaniam et al. [11] to identify the scattering albedo and the asymmetry factor of the phase function in an anisotropically scattering medium. However, the identification of absorption and scattering coefficients was impossible in $[10,11]$ as the analysis required the knowledge of the optical thickness. Galtier et al. [12] circumvented this difficulty by the use of null-collisions method [13] to express a radiative quantity as a polynomial of absorption and scattering coefficients.

In this work, SMC algorithms presented in [12] are developed within the frame of inverse radiative transfer. A complete SMC framework for the identification of absorption and scattering coefficients of heterogeneous semitransparent materials from measurements of directional-hemispherical transmittance and reflectance is proposed. An inverse analysis based on SMC is developed and advantages related to the use of SMC are highlighted: from inverse analysis (discussion on the well or ill-posed character of the problem), up to the fast 
experimental identification of radiative properties, including experimental and numerical errors. The whole method is illustrated here in the case of a low density fibrous medium but the approach is not restricted to this material and can be used for other types of heterogeneous media.

The paper is structured as follows. In the second section, experimental setup and spectroscopic measurements are presented. In the third section, details of the proposed Symbolic approach are given: SMC algorithm that allows to obtain simple polynomial forms of directional-hemispherical transmittance and reflectance as function of absorption and scattering coefficients is presented. These polynomials are then used to determine the nature (well or ill-posed) of the inverse problem, including experimental and numerical errors in the analysis. Finally, in the last section, inversion based on these polynomials is carried out to retrieve absorption and scattering coefficients of a Quartzel sample.

\section{Sample description and experimental measurements}

\subsection{Sample description}

The medium considered is a Quartzel low density felt (Saint-Gobain Quartz) sample. It is used as insulation material for aircraft engines or furnace closures. It is also used as a support for catalysts in domestic and industrial catalytic heaters. Felts provided by Saint-Gobain have an estimated thickness of $11 \mathrm{~mm}$ when not compressed, which corresponds, according to the material brochure, to an areal weight of $100 \mathrm{~g} \cdot \mathrm{m}^{-2}$ and an estimated density of $[10 ; 20] \mathrm{kg}^{-\mathrm{m}^{-3}}$. Low density felts are produced from 9 microns pure fused quartz fibers (Quartzel wool) with a constant diameter along their length. They are randomly oriented in parallel planes and are impregnated with an organic binder (PolyVinyl Alcohol). In this work, anisotropy of the sample was not assessed as bi-directional measurements of transmittance and reflectance were not performed. Size distribution of fibers in the medium can be modeled as a normal distribution with $9 \mu \mathrm{m}$ mean and $2 \mu \mathrm{m}$ variance. Porosity of the studied sample is higher than 
$95 \%$ (volume fraction of fibers lower than $5 \%$ ). A picture of the felt is shown in Figure 1.

The studied sample is cylindrical with $24 \mathrm{~mm}$ diameter and $4 \mathrm{~mm}$ width. The sample is constrained between two $\mathrm{ZnSe}$ windows with a multilayer Broad Band Anti Reflectance coating in the spectral region $[830 ; 3330] \mathrm{cm}^{-1}$, all in a 3D-printed sample holder specifically designed for the current apparatus.

\subsection{Experimental setup, measurements and uncertainty evaluation}

Spectroscopic measurements were carried out using a Bruker IFS 66v/S Fourier-transform infrared (FTIR) spectrometer. The incident radiative flux is provided by a Globar source for mid-infrared (MIR) spectroscopy (spectral range of $[666 ; 5000] \mathrm{cm}^{-1}$ equivalent to $\left.[2 ; 15] \mu \mathrm{m}\right)$. An infragold A562G integrating sphere provided with a DTGS detector allows directional-hemispherical transmittance and reflectance measurements as illustrated in figure 2. The total diameter of the sphere is $75 \mathrm{~mm}$ and the inner wall of the sphere is coated with a layer of diffuse reflecting gold that reflects the incoming light several times and scatters it uniformly around the interior of the sphere (the integrating sphere ensures a homogeneous spatial light intensity distribution). The apparatus is permanently flushed with dry air with low $\mathrm{CO}_{2}$ and $\mathrm{H}_{2} \mathrm{O}$ concentration to reduce atmospheric absorption in the following spectral ranges: $[3389 ; 4000] \mathrm{cm}^{-1}$, $[2222 ; 2398] \mathrm{cm}^{-1},[1250 ; 2083] \mathrm{cm}^{-1}$ and $[581 ; 757] \mathrm{cm}^{-1}$. Purging the integrating sphere also ensures a constant concentration of atmospheric constituents over time.

Experimental transmittance and reflectance spectra for the sample described above are provided as a function of wavenumbers $\eta\left[\mathrm{cm}^{-1}\right]$ and wavelengths $\lambda=10^{4} / \eta[\mu m]$ in figure 3 . Measurements of transmittance and reflectance are performed, at ambient temperature in the spectral range $[700 ; 3700] \mathrm{cm}^{-1}$. One spectrum is the result of 50 scans of the interferometer at a spectral resolution of $16 \mathrm{~cm}^{-1}$, and measurements illustrated in figure 3 are obtained by averaging 100 spectra performed on the same sample over a week period. Transmittance (respectively reflectance) is the ratio of transmitted (respectively reflected) ra- 
diative heat flux by the sample to the incident one called reference. Spectra are obtained from two consecutive measurements. Because it is necessary to perform these two successive steps, atmospheric conditions vary between the two measurements. Despite all precautions to reduce effects of $\mathrm{CO}_{2}$ absorption, its concentration still varied during time which induced absorption bands mainly near $\eta=2350 \mathrm{~cm}^{-1}$ (see colored band in figure 3 ). Transmittance and reflectance measurements in this absorption band will not be taken into account in the identification process.

In order to estimate the uncertainty of FTIR spectroscopic measurements, one must take into account a large number of sources that may affect the interpretation of the spectrum. Sources of errors can be a change of ambient temperature, a variation of the concentration of atmospheric absorbents like water vapor and carbon dioxyde, an inefficient cooling of the MIR source, a heating of the source's aperture, a loss of efficiency of optics, a non-linear response of detector, interreflections involving the sample, etc. These errors lead to experimental uncertainties. Measurement uncertainty is defined as:

$$
U=k \sqrt{\sum_{i=1}^{i=n} u_{b, i}^{2}+u_{\text {rand }}^{2}}
$$

where $u_{b, i}$ are individual sources of uncertainty and $u_{\text {rand }}$ is the uncertainty related to random errors. The uncertainties are wavelength-dependent, therefore they must be computed for the specific wavelength and the specific sample. Most of errors are dependent and it is difficult to quantify their associated uncertainties. A first approach to estimate the measurements uncertainties is to only take into account the effects of the detector internal noise $\left(u_{b}\right)$ and to consider that all other error sources are random $\left(u_{\text {rand }}\right)$. In figures $3 a$ and $3 \mathrm{~b}$, error bars were obtained for each wavenumber using the detector internal noise and the standard-deviation of 100 measurements performed over a week period. The coverage factor $k$ was taken equal to 2 in order to have an extended absolute uncertainty with a confidence interval of 95\%. A quasi-similar approach was proposed by [14] for the evaluation of emissivity from transmittance and 
reflectance measurements at ambient temperature. In the present work, absolute experimental uncertainties were estimated at 0.01 .

\section{Identification of absorption and scattering coefficients based on SMC Analysis}

\subsection{Model}

A schematic representation of the problem considered is given in figure 4. The medium (Quartzel sample) noted $\Delta$ is at ambient temperature. An incident beam of intensity $I_{0, \eta}$ in the $\boldsymbol{u}_{\mathbf{0}}$ direction crosses the surface $S_{0}$. We define the surface $S_{R}$ (respectively $S_{T}$ ) corresponding to the abscissa $x=0 \mathrm{~mm}$ (respectively $x=4 \mathrm{~mm}$ ) for which spectral reflectance (respectively transmittance) is estimated.

The Radiative Transfer Equation (RTE) in an equivalent homogeneous absorbing and scattering medium is considered for modeling radiative transfer in the fibrous material. Bidirectional measurements need to be performed if information about anisotropy is expected. Consequently, phase function cannot be identified from directional-hemispherical transmittance and reflectance, and may be assumed either isotropic or modeled by the Delta-Eddington (DE) approximation. In DE case, the phase function asymmetry factor $g_{\eta}$ is included in the reduced scattering coefficient $\sigma_{\eta}^{\prime}=\sigma_{\eta}\left(1-g_{\eta}\right)$ where $\sigma_{\eta}$ is the scattering coefficient.

RTE in homogeneous media has been chosen to model radiative transfer in Quartzel sample because of its low density, its high porosity and the random orientation of its fibers. The boundary conditions are given by $I_{\eta}\left(\mathbf{x}_{0}, \mathbf{u}_{0}\right)=$ $I_{0, \eta}$ if $\mathbf{x}_{0} \in S_{0}, 0$ elsewhere. Using this model, absorption coefficient $\kappa_{\eta}$ and scattering coefficient $\sigma_{\eta}$ need to be identified.

\subsection{Standard forward null-collision Monte Carlo algorithm}

A forward null-collision Monte Carlo algorithm is applied to simulate radiative transfer in the medium. Null-collision algorithms [13] are needed to express 
the radiative intensity as a polynomial of $\kappa_{\eta}$ and $\sigma_{\eta}$ as shown in [12]. The formulations, based on the integral form of the RTE, of transmitted directionalhemispherical transmittance $T_{\eta}$ and reflectance $R_{\eta}$ are given by the following equations:

$$
\begin{aligned}
T_{\eta} & =\frac{\int_{S_{0}} I_{0, \eta} d S \times \Gamma_{\eta}\left(\left(\boldsymbol{x}_{\mathbf{0}}, \boldsymbol{u}_{\mathbf{0}}\right) \rightarrow\left(\boldsymbol{x}_{\boldsymbol{T}}, \boldsymbol{u}_{\boldsymbol{T}}\right)\right)}{\int_{S_{0}} I_{0, \eta} d S} \\
R_{\eta} & =\frac{\int_{S_{0}} I_{0, \eta} d S \times \Gamma_{\eta}\left(\left(\boldsymbol{x}_{\mathbf{0}}, \boldsymbol{u}_{\mathbf{0}}\right) \rightarrow\left(\boldsymbol{x}_{\boldsymbol{R}}, \boldsymbol{u}_{\boldsymbol{R}}\right)\right)}{\int_{S_{0}} I_{0, \eta} d S}
\end{aligned}
$$

where $\boldsymbol{x}_{\boldsymbol{T}}\left(\right.$ or $\left.\boldsymbol{x}_{\boldsymbol{R}}\right)$ is a position on the surface $S_{T}\left(\right.$ or $\left.S_{R}\right)$ and $\boldsymbol{u}_{\boldsymbol{T}}\left(\right.$ or $\left.\boldsymbol{u}_{\boldsymbol{R}}\right)$ is the optical path direction when it outgoes the surface $S_{T}$ (or $S_{R}$ ). $\Gamma$ is a dimensionless quantity and can be interpreted as a transmission function from the incident beam to the outbound surface. The determination of the transmission function $\Gamma_{\eta}$ is based on a forward Monte Carlo algorithm and is estimated recursively using the following expression:

$$
\begin{aligned}
& \Gamma_{\eta}\left(\left(\boldsymbol{x}_{\mathbf{0}}, \boldsymbol{u}_{\mathbf{0}}\right) \rightarrow\left(\boldsymbol{x}_{\boldsymbol{T}}, \boldsymbol{u}_{\boldsymbol{T}}\right)\right) \\
& =\int_{0}^{\infty} \widehat{\beta}_{\eta} \exp \left(-\widehat{\beta}_{\eta} l_{1}\right) d l_{1} \times\left(H ( \boldsymbol { x } _ { \mathbf { 1 } } \in \Delta ) \left[\frac{\kappa_{\eta}}{\widehat{\beta}_{\eta}} \times 0+\frac{\sigma_{\eta}}{\widehat{\beta}_{\eta}} \int_{4 \pi} \frac{1}{4 \pi} \Gamma_{\eta}\left(\boldsymbol{x}_{\mathbf{1}}, \boldsymbol{u}_{\mathbf{1}}\right) d \boldsymbol{u}_{\mathbf{1}}\right.\right. \\
& \left.\left.\quad+\frac{\widehat{\beta}_{\eta}-\kappa_{\eta}-\sigma_{\eta}}{\widehat{\beta}_{\eta}} \Gamma_{\eta}\left(\boldsymbol{x}_{\mathbf{1}}, \boldsymbol{u}_{\mathbf{1}}=\boldsymbol{u}_{\mathbf{0}}\right)\right]+H\left(\boldsymbol{x}_{\mathbf{1}} \notin \Delta\right) H\left(\boldsymbol{x}_{\mathbf{1}} \rightarrow S_{T}\right)\right)
\end{aligned}
$$

where $H(C)$ is the Heaviside function (equal to 1 if condition $C$ is satisfied), $\widehat{\beta}_{\eta}=\kappa_{\eta}+\sigma_{\eta}+\gamma_{\eta}$ is the extinction coefficient including null-collisions [13] and $\gamma_{\eta}$ is the null-collision coefficient. This expression is also valid for reflectance by changing index $T$ with $R$.

Standard forward null-collisions Monte-Carlo algorithms perform a large number $N_{M C}$ of independent optical path realizations indexed $i$. Free-paths are sampled according to the probability density $\widehat{p}_{L}(l)=\widehat{\beta}_{\eta} \exp \left(-\widehat{\beta}_{\eta} l\right)$. Nullcollisions coefficient $\gamma_{\eta}$ introduces pure-forward scattering events. The value of 
$\widehat{\beta}_{\eta}$ determines the upper bound of the real extinction coefficient $\beta_{\eta}=\kappa_{\eta}+\sigma_{\eta}$ over which the functional will be defined [13].

Using this algorithm, the $i$-th optical path realization starts with the sampling of a location $\boldsymbol{x}_{\mathbf{0}}$ in $S_{0}$ according to a uniform probability density function $\frac{1}{S_{0}}$. A free path $l_{1, i}$ is then sampled according to $\widehat{p}_{L}\left(l_{1, i}\right)$ and a new position $\boldsymbol{x}_{1, i}=\boldsymbol{x}_{\mathbf{0}}+l_{1, i} \boldsymbol{u}_{\mathbf{0}}$ is deduced. At this point, two approaches can be used for the standard algorithm.

In the most usual approach, a step is required to statistically determine which type of event occurs: an absorption with probability given by $\frac{\kappa_{\eta}}{\widehat{\beta}_{\eta}}$, a scattering given by the probability $\frac{\sigma_{\eta}}{\widehat{\beta}_{\eta}}$ or a null-collision with probability $\frac{\widehat{\beta}_{\eta}-\kappa_{\eta}-\sigma_{\eta}}{\widehat{\beta}_{\eta}}$. However, an absorption event will always lead to a null Monte Carlo weight since the quantities of interest are transmittance and reflectance, not absorptance (Eq 3). Therefore, an energy partitioning approach is applied in this work as in [15]. Following this approach, absorption attenuation is still accounted for in the sampling of extinction free paths according to Beer's law $\widehat{\beta} \exp (-\widehat{\beta} L)$, but the statistical determination of a collision will only concern scattering and nullcollision. A probability $P_{s, \eta}$ for scattering, and $P_{n, \eta}=1-P_{s, \eta}$ for null-collision, is therefore introduced in the algorithm. Eq 3 becomes:

$$
\begin{aligned}
& \Gamma_{\eta}\left(\left(\boldsymbol{x}_{\mathbf{0}}, \boldsymbol{u}_{\mathbf{0}}\right) \rightarrow\left(\boldsymbol{x}_{\boldsymbol{T}}, \boldsymbol{u}_{\boldsymbol{T}}\right)\right) \\
& =\int_{0}^{\infty} \widehat{\beta}_{\eta} \exp \left(-\widehat{\beta}_{\eta} l_{1}\right) d l_{1} \times\left(H ( \boldsymbol { x } _ { \mathbf { 1 } } \in \Delta ) \left[P_{s, \eta} \frac{\sigma_{\eta}}{\widehat{\beta}_{\eta} P_{s, \eta}} \int_{4 \pi} \frac{1}{4 \pi} \Gamma_{\eta}\left(\boldsymbol{x}_{\mathbf{1}}, \boldsymbol{u}_{\mathbf{1}}\right) d \boldsymbol{u}_{\mathbf{1}}\right.\right. \\
& \left.\left.\quad+\left(1-P_{s, \eta}\right) \frac{\widehat{\beta}_{\eta}-\kappa_{\eta}-\sigma_{\eta}}{\widehat{\beta}_{\eta}\left(1-P_{s, \eta}\right)} \Gamma_{\eta}\left(\boldsymbol{x}_{\mathbf{1}}, \boldsymbol{u}_{\mathbf{1}}=\boldsymbol{u}_{\mathbf{0}}\right)\right]+H\left(\boldsymbol{x}_{\mathbf{1}} \notin \Delta\right) H\left(\boldsymbol{x}_{\mathbf{1}} \rightarrow S_{T}\right)\right)
\end{aligned}
$$

where $P_{s, \eta}$ can be expressed as:

$$
P_{s, \eta}=\frac{\sigma_{\eta}}{\sigma_{\eta}+\gamma_{\eta}}=\frac{\sigma_{\eta}}{\widehat{\beta}_{\eta}-\kappa_{\eta}}
$$

Consequently, at position $\boldsymbol{x}_{1, i}$, scattering and null-collision events are sampled according to $P_{s, \eta}$ and $1-P_{s, \eta}$. If scattering occurs at position $\boldsymbol{x}_{1, i}$, a new 
direction of propagation $\boldsymbol{u}_{\mathbf{1}}$ is sampled according to $\frac{1}{4 \pi}$, and the transmission function $\Gamma$ is multiplied by $\frac{\sigma_{\eta}}{\widehat{\beta}_{\eta} P_{s, \eta}}$. If a null-collision occurs, direction of propagation $\boldsymbol{u}_{\mathbf{1}}$ is equal to the previous one $\boldsymbol{u}_{\mathbf{0}}$ as null-collisions correspond to pure forward-scattering, and the transmission function $\Gamma$ is multiplied by $\frac{\widehat{\beta}_{\eta}-\kappa_{\eta}-\sigma_{\eta}}{\widehat{\beta}_{\eta}\left(1-P_{s, \eta}\right)}$. A new free path $l_{2, i}$ is then sampled. The algorithm loops until the optical path exits the medium. If the optical path exits the domain through the surface $S_{T}$, transmittance is implemented (the associated Heaviside distribution corresponding to the estimation of directional-hemispherical transmittance is $H_{T, i}=1$, and $\left.H_{R, i}=0\right)$. On the opposite, if the outbound surface is $S_{R}$, reflectance is implemented (the associated Heaviside distribution corresponding to the estimation of directional-hemispherical reflectance is $H_{R, i}=1$, and $\left.H_{T, i}=0\right)$.

For illustration, let us assume that along the $i$-th Monte Carlo path sample, the following events occur: one scattering, two null-collisions and another scattering before the optical path exits the medium. According to equation 4 and using the energy partitioning approach, the associated Monte Carlo weight for the estimation of transmittance is expressed in this case by:

$$
w_{T, i}=\frac{\sigma_{\eta}}{\widehat{\beta}_{\eta} P_{s, \eta}}\left\{\frac{\widehat{\beta}_{\eta}-\kappa_{\eta}-\sigma_{\eta}}{\widehat{\beta}_{\eta}\left(1-P_{s, \eta}\right)}\left[\frac{\widehat{\beta}_{\eta}-\kappa_{\eta}-\sigma_{\eta}}{\widehat{\beta}_{\eta}\left(1-P_{s, \eta}\right)}\left(\frac{\sigma_{\eta}}{\widehat{\beta}_{\eta} P_{s, \eta}} H_{T, i}\right)\right]\right\}
$$

which, given the expression of $P_{s, \eta}$ (Eq. 5), simplifies into:

$$
w_{T, i}=\left(\frac{\widehat{\beta_{\eta}}-\kappa_{\eta}}{\widehat{\beta_{\eta}}}\right)^{4} H_{T, i}
$$

In general, Monte Carlo weights for the estimation of transmittance and reflectance can be expressed as:

$$
w_{T, i}=\left(\frac{\widehat{\beta_{\eta}}-\kappa_{\eta}}{\widehat{\beta_{\eta}}}\right)^{N_{s c a, i}+N_{n c, i}} H_{T, i}
$$

where $N_{s c a, i}$ and $N_{n c, i}$ are the number of scattering and null-collision events that occurred along the $i$-th optical path. 


\subsection{Symbolic Monte Carlo algorithm}

Radiative quantities can be expressed using SMC as bivariate polynomials of absorption coefficient $\kappa_{\eta}$ and scattering coefficient $\sigma_{\eta}[12]$ :

$$
\begin{aligned}
& T_{\eta}\left(\kappa_{\eta}, \sigma_{\eta}\right)=\sum_{j=0}^{\infty} \sum_{k=0}^{\infty} a_{j k} \kappa_{\eta}^{j} \sigma_{\eta}^{k} \\
& R_{\eta}\left(\kappa_{\eta}, \sigma_{\eta}\right)=\sum_{j=0}^{\infty} \sum_{k=0}^{\infty} b_{j k} \kappa_{\eta}^{j} \sigma_{\eta}^{k}
\end{aligned}
$$

The standard forward null-collision Monte Carlo algorithm described in previous section can be applied only if numerical values are affected to the absorption and scattering coefficients. In SMC, if $\kappa_{\eta}$ and $\sigma_{\eta}$ are kept under their symbolic form, and consequently the probability of scattering event $P_{s, \eta}=\frac{\sigma_{\eta}}{\widehat{\beta}_{\eta}-\kappa_{\eta}}$ (and $1-P_{s, \eta}$ for null-collision) is unknown. The choice of $P_{s, \eta}$ becomes therefore arbitrary [12] (this arbitrary probability is denoted $\widetilde{P_{s}}$ ). In this work, $\widetilde{P_{s}}$ is chosen equal to 0.5 for all wavenumbers. The choice of this arbitrary probability has an impact on the variance which is analyzed in Appendix A.

Now, if we assume that along the $i$-th Monte Carlo path sample, one scattering, two null-collisions and another scattering events occur before the optical path exits the medium (i.e., $N_{n c, i}=2$ and $N_{s c a, i}=2$ ), the associated Monte Carlo weight is expressed by:

$$
w_{S M C, T, i}=\frac{\sigma_{\eta}}{\widehat{\beta}_{\eta} \widetilde{P}_{s}}\left\{\frac{\widehat{\beta}_{\eta}-\kappa_{\eta}-\sigma_{\eta}}{\widehat{\beta}_{\eta}\left(1-\widetilde{P}_{s}\right)}\left[\frac{\widehat{\beta}_{\eta}-\kappa_{\eta}-\sigma_{\eta}}{\widehat{\beta}_{\eta}\left(1-\widetilde{P}_{s}\right)}\left(\frac{\sigma_{\eta}}{\widehat{\beta}_{\eta} \widetilde{P}_{s}} H_{T, i}\right)\right]\right\}
$$

which can be rewritten as a polynomial:

$$
w_{S M C, T, i}=\left(\frac{\sigma_{\eta}}{\widehat{\beta}_{\eta} \widetilde{P}_{s}}\right)^{2}\left(\frac{\widehat{\beta}_{\eta}-\kappa_{\eta}-\sigma_{\eta}}{\widehat{\beta}_{\eta}\left(1-\widetilde{P}_{s}\right)}\right)^{2} H_{T, i}
$$

More generally, the total contribution of the $i$-th optical path with $N_{n c, i}$ nullcollision events and $N_{s c a, i}$ scattering events is written in the general case:

$$
w_{S M C, T, i}=\left(\frac{\widehat{\beta}_{\eta}-\kappa_{\eta}-\sigma_{\eta}}{\widehat{\beta}_{\eta}\left(1-\widetilde{P}_{s}\right)}\right)^{N_{n c, i}}\left(\frac{\sigma_{\eta}}{\widehat{\beta}_{\eta} \widetilde{P}_{s}}\right)^{N_{s c a, i}} H_{T, i}
$$


In order to introduce the symbolic MC weight $a_{j k, i}$ of the polynomial coefficients (associated to the $i$-th optical path), Eq. 12 is rewritten as:

$$
w_{S M C, T, i}=\sum_{j=0}^{\infty} \sum_{k=0}^{\infty}\left(\frac{\widehat{\beta}_{\eta}-\kappa_{\eta}-\sigma_{\eta}}{\widehat{\beta}_{\eta}\left(1-\widetilde{P}_{s}\right)}\right)^{j}\left(\frac{\sigma_{\eta}}{\widehat{\beta}_{\eta} \widetilde{P}_{s}}\right)^{k} a_{j k, i}
$$

where $a_{j k, i}$ is a non-zero coefficient only if $j=N_{n c, i}$ and $k=N_{s c a, i}$ :

$$
a_{j k, i}=\delta_{j, N_{n c, i}} \delta_{k, N_{s c a, i}} H_{T, i}
$$

32

33 according to:

$$
\begin{aligned}
& T_{\eta} \simeq \sum_{j=0}^{\infty} \sum_{k=0}^{\infty} \overline{a_{j k}}\left(\frac{\widehat{\beta}_{\eta}-\kappa_{\eta}-\sigma_{\eta}}{\widehat{\beta}_{\eta}\left(1-\widetilde{P}_{s}\right)}\right)^{j}\left(\frac{\sigma_{\eta}}{\widehat{\beta}_{\eta} \widetilde{P}_{s}}\right)^{k} \\
& R_{\eta} \simeq \sum_{j=0}^{\infty} \sum_{k=0}^{\infty} \overline{b_{j k}}\left(\frac{\widehat{\beta}_{\eta}-\kappa_{\eta}-\sigma_{\eta}}{\widehat{\beta}_{\eta}\left(1-\widetilde{P}_{s}\right)}\right)^{j}\left(\frac{\sigma_{\eta}}{\widehat{\beta}_{\eta} \widetilde{P}_{s}}\right)^{k}
\end{aligned}
$$

$$
\begin{aligned}
& \overline{a_{j k}} \approx \frac{1}{N_{M C}} \sum_{i=1}^{N_{M C}} a_{j k, i} \\
& \overline{b_{j k}} \approx \frac{1}{N_{M C}} \sum_{i=1}^{N_{M C}} b_{j k, i}
\end{aligned}
$$

A flowchart of the SMC algorithm is shown in figure 5. At each optical path $i \in\left\{1, \ldots, N_{M C}\right\}$, the number of scattering $N_{s c a, i}$ and null-collisions events $N_{n c, i}$ is counted in order to calculate the coefficients $a_{j k, i}$ and $b_{j k, i}$ according to Eq. 14. Once the $N_{M C}$ optical paths have been generated, the coefficients $\overline{a_{j k}}$ and $\overline{b_{j k}}$ are estimated according to Eq. 16.

$R_{\eta}$ and $T_{\eta}$ are expressed as polynomial functions of $\sigma_{\eta}$ and $\widehat{\beta}-\kappa_{\eta}-\sigma_{\eta}$, but it is possible to re-express $R_{\eta}$ and $T_{\eta}$ as a polynomials of $\kappa_{\eta}$ and $\sigma_{\eta}$ since the value of $\widehat{\beta}$ is fixed in the SMC procedure. 
It should be emphasized that all the estimated polynomial coefficients $\overline{a_{j k}}$ and $\overline{b_{j k}}$ are independent of the wavenumbers. This is a direct consequence of the choice of a constant arbitrary probability $\widetilde{P_{s}}$. If $\widetilde{P_{s}}$ was wavenumber-dependent, as many polynomials as wavenumbers would be needed. Here, one single SMC calculation is sufficient to express $T_{\eta}$ and $R_{\eta}$ as functions of $\kappa_{\eta}$ and $\sigma_{\eta}$, and only those two polynomials are used over the full spectrum.

\subsection{Inverse analysis based on SMC polynomials}

All results presented in the following subsections were obtained using $N_{M C}=$ $10^{6}, \widehat{\beta_{\eta}}=20 \mathrm{~cm}^{-1}$ and $\widetilde{P_{s}}=0.5 . S_{0}$ is a disk with $12 \mathrm{~mm}$ diameter. Without any a priori information about scattering inside the considered material, the choice of $\widetilde{P s}=0.5$ represents a good compromise to obtain a satisfactory accuracy for both thin and thick scattering optical thickness (as discussed in [12]). Here, with $\widetilde{P_{s}}=0.5$, the average relative standard deviation is $1.1 \%$ for transmittance and $1.0 \%$ for reflectance (maximum relative standard-deviation is $2.1 \%$ for transmittance and $2.6 \%$ for reflectance) and allows the identification of radiative properties in most part of the spectrum. The influence of the choice of $\widetilde{P_{s}}=0.5$ on the standard-deviation is discussed in Appendix A.

The functional obtained with SMC allows to efficiently estimate the quantities (since one simulation is required), $T_{\eta}$ and $R_{\eta}$, all over the parameter space: $\kappa_{\eta} \in[0,10]$ and $\sigma_{\eta} \in[0,10]$. It is therefore possible to plot isovalues of transmittance and reflectance as functions of absorption and scattering coefficients as depicted in Figure 6.

According to the RTE model, isolines that lead to given values of $T_{\eta}$ and $R_{\eta}$ are displayed for an infinity of couples $\left(\kappa_{\eta}, \sigma_{\eta}\right)$. An isoline gives the range of possible values of $\kappa_{\eta}$ and $\sigma_{\eta}$ that reproduce the measured transmittance or reflectance. For example, the isoline $T_{\eta}=0.3$ in figure 6 a gives the possible values of absorption and scattering coefficients $\kappa_{\eta} \in[0 ; 3] \mathrm{cm}^{-1}$ and $\sigma_{\eta} \in[0 ; 8.7]$ $\mathrm{cm}^{-1}$ that are compatible with this particular value of $T_{\eta}$.

From those isolines, it is possible to determine if the solution of the inverse problem exists, and if it is unique. The first case considered in figure 7a cor- 
responds to the measured values $T_{\eta}=0.03$ and $R_{\eta}=0.18$ at $\eta=900 \mathrm{~cm}^{-1}$. In figure $7 \mathrm{a}$, the isoline corresponding to $T_{\eta}=0.03$ is depicted in red lines, and the isoline corresponding to $R_{\eta}=0.18$ is depicted in green lines. This graphical method, also called the contour intersection approach [16], allows to determine the absorption and scattering coefficients that are solutions of the inverse problem by plotting the isolines corresponding to the measurements of $T_{\eta}$ and $R_{\eta}$. Intersections represent values of $\kappa_{\eta}$ and $\sigma_{\eta}$ that reproduce the measured transmittance and reflectance according to the RTE model. If no intersection appears, then the solution of the inverse problem does not exist. If several intersections are observed, then the solution exists, but is not unique. In figure $7 \mathrm{a}$, only one intersection is obtained in the parameter space, which shows that the solution exists and is unique: $\kappa_{\eta}=3.90 \mathrm{~cm}^{-1}$ and $\sigma_{\eta}=7.44$ $\mathrm{cm}^{-1}$. The same conclusion can be drawn in figure $7 \mathrm{~b}$ where measured values $T_{\eta}=0.01$ and $R_{\eta}=0.08$ at $\eta=1189 \mathrm{~cm}^{-1}$ are considered: $\kappa_{\eta}=7.62 \mathrm{~cm}^{-1}$ and $\sigma_{\eta}=4.92 \mathrm{~cm}^{-1}$.

However, even if the solution exists and is unique, the inverse problem can be ill-posed if the solution is unstable according to Hadamard's definition. Consequently, the feasibility of the identification cannot be ensured as long as experimental and numerical errors are not taken into account. Using SMC polynomials, experimental and numerical errors can be easily included in the analysis as illustrated in figure 7. Measurement and numerical (SMC standard-deviations) errors are introduced by plotting the isolines corresponding to $T_{\eta}+\varepsilon\left(T_{\eta}\right), T_{\eta}-\varepsilon\left(T_{\eta}\right), R_{\eta}+\varepsilon\left(R_{\eta}\right)$ and $R_{\eta}-\varepsilon\left(R_{\eta}\right)$ where $\varepsilon$ is the total error at wavenumber $\eta$. The colored (orange) polygons and filled plots in figure 7 represent the range of possible $\kappa_{\eta}$ and $\sigma_{\eta}$ that reproduce the measured transmittance and reflectance according to the RTE model, with respect to the estimated experimental and numerical uncertainties.

In figure $7 \mathrm{a}$, the estimated relative uncertainties of $30 \%$ for transmittance and $4 \%$ for reflectance are taken into account at $\eta=900 \mathrm{~cm}^{-1}$. Absorption coefficients range in the intervals $[3.46 ; 4.50] \mathrm{cm}^{-1}$ (relative error lower than $15.4 \%$ ) and scattering coefficients range in $[6.57 ; 8.61] \mathrm{cm}^{-1}$ (relative error lower 
than $15.8 \%$ ). There may be noise amplification, but the domain within which the couple $\left(\kappa_{\eta}, \sigma_{\eta}\right)$ is located remains of limited size. In figure $7 \mathrm{~b}$, errors are taken into account at $\eta=1189 \mathrm{~cm}^{-1}$ (98\% for transmittance and $10 \%$ for reflectance). Possible solutions of the inverse problem are not bounded as all absorption and scattering coefficients that are respectively greater than 6.25 $\mathrm{cm}^{-1}$ and $3.78 \mathrm{~cm}^{-1}$ (filled bold polygon) belong to the parameter space region where transmittance is $0.01 \pm 98 \%$ and reflectance is $0.09 \pm 9.4 \%$ according to the RTE model. In this case, identification is not possible; others measurements and/or another radiative models are needed to ensure the well-posed character of the inverse problem.

These examples illustrate how SMC allows to determine the existence, unicity and stability of the inverse problem solution.

\subsection{Application to a Quartzel sample}

Once the spectral range where the absorption and scattering coefficients identification is feasible has been determined from SMC analysis, the inverse procedure can be applied. Identification of $\kappa_{\eta}$ and $\sigma_{\eta}$ at a wavenumber $\eta$ is performed from measurements of transmittance and reflectance and from SMC polynomials. Here, a Levenberg-Marquardt algorithm is used to determine $\kappa_{\eta}$ and $\sigma_{\eta}$.

In the inverse procedure, the two polynomials corresponding to transmittance and reflectance are used as direct model for radiation, and avoid the costly resolution of the RTE at each wavenumber, and each iteration of the inversion algorithm. Thus, only one SMC simulation is carried out during the inverse method, which decreases significantly the computational cost.

Identified absorption and scattering coefficients are plotted in figure 8. Error bars of identified $\kappa_{\eta}$ and $\sigma_{\eta}$ in figure 8 are determined based on the principle described in figure 7 . They correspond to the minimum and maximum value of the admissible zone with an experimental uncertainty of \pm 0.01 which is the maximal value of calculated experimental uncertainty over the whole spectrum. SMC analysis showed that the identification of radiative properties is impossible 
for wavenumbers in the intervals $[700 ; 870]$ and $[950 ; 1245] \mathrm{cm}^{-1}$. These spectral regions are depicted in grey in figure 8. In these regions, measured transmittance is lower than 0.01. It ranges within the detector internal noise which leads to a relative uncertainty of $100 \%$. In this particular case, the range of possible absorption and scattering coefficients that are solutions of the inverse problem are unbounded as it was illustrated in figure 7b. The medium is optically thick and the RTE model turns out to be unsuitable for the identification at these wavenumbers. SMC showed that the identification of radiative properties is possible in other spectral regions i.e., [870;950] and $[1245 ; 3700] \mathrm{cm}^{-1}$.

\section{Conclusion}

In the present work, SMC was applied to express directional-hemispherical transmittance and reflectance as polynomials of absorption and scattering coefficients. An analysis based on these polynomials allows to determine if the radiative properties identification is feasible or not, illustrating the well or illposed nature of the inverse problem. Indeed, the contour intersection approach shows if the solution exists and is unique, and an analysis based on experimental and numerical uncertainties shows if the solution is reliable or not. When identification is feasible, SMC polynomials combined with an optimization algorithm allows to retrieve the range of absorption and scattering coefficients that are solutions of the inverse problem. The approach has been applied to identify the radiative properties of a heterogeneous material, a Quartzel low-density felt. Using the SMC polynomials as direct model in the inverse iterative procedure, the RTE is only solved once with SMC. The numerical efficiency is therefore significantly improved.

The method proposed in this paper can be applied to other kind of materials with different structure, density and porosity to identify potential limits of SMC. Moreover, it can also be applied to phase function parameters identification if bidirectional measurements are carried out. Indeed, SMC can be used to express radiative quantities as polynomials of absorption and scattering coefficients, as 
364

well as phase function parameters [17]. The SMC polynomials would then allow the identification of phase function (in addition to absorption and scattering coefficients).

In future works, the identification of radiative properties of materials at high temperature from spectroscopic emission measurements will be investigated.

The experimental bench described in [18] will be used. The development of an efficient identification strategy at high temperature remains a challenging task since the ill-posed character of the inverse problem has been highlighted in [19] when the RTE model is chosen. The SMC framework proposed in this work will be developed further in order to guide inverse modeling in this context. 


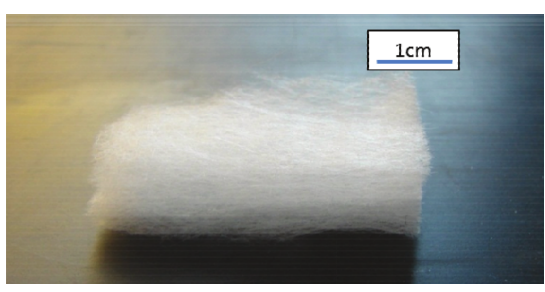

(a)

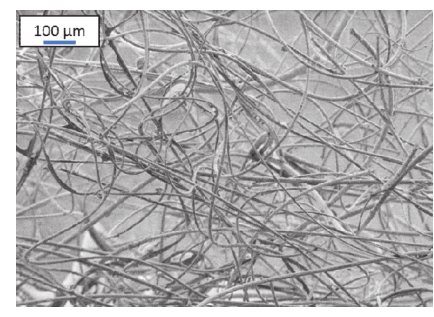

(b)

Figure 1: (a) Example of a Quartzel sample. (b) Scanning Electron Microscope (SEM) picture of Quartzel. Pictures from [20] 

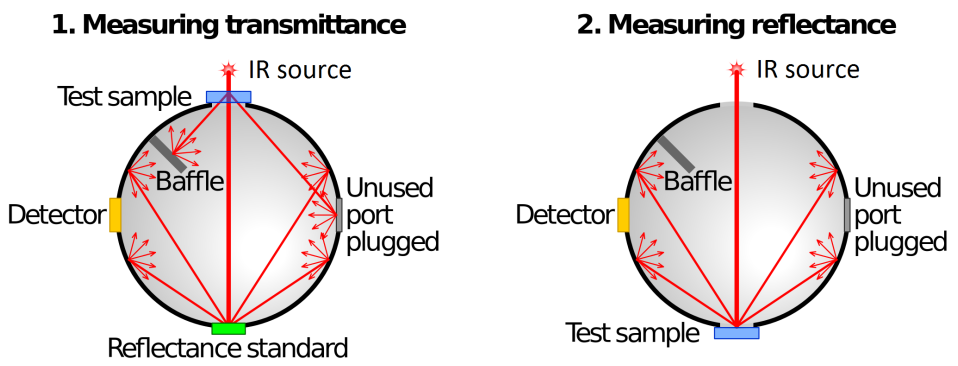

Figure 2: Integrating sphere configurations for transmittance and reflectance 


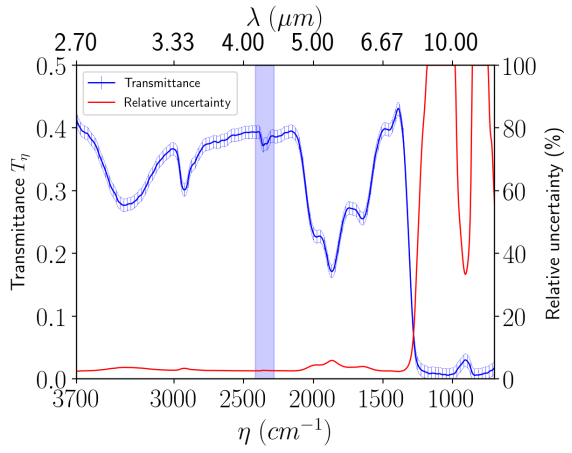

(a)

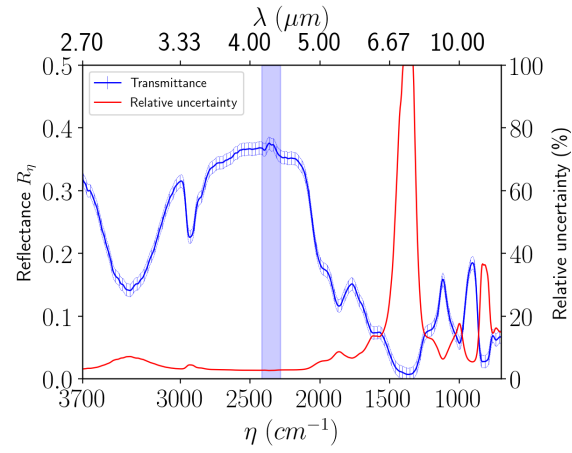

(b)

Figure 3: Mean measurements of (a) transmittance and (b) reflectance with their associated uncertainties. The colored blue band corresponds to $\mathrm{CO}_{2}$ absorption band between $\eta=2222$ and $\eta=2398 \mathrm{~cm}^{-1}$ where measurements are not taken into account. 


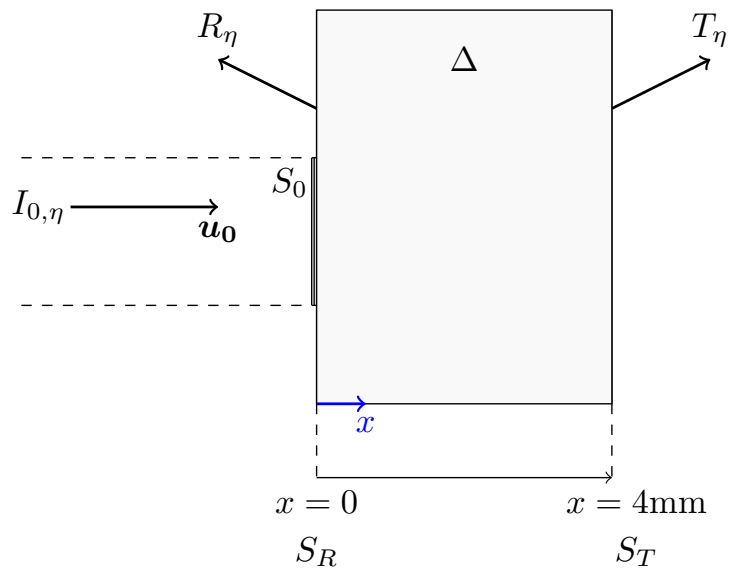

Figure 4: Representation of the sample in a configuration of directional-hemispherical measurement of transmittance and reflectance. 


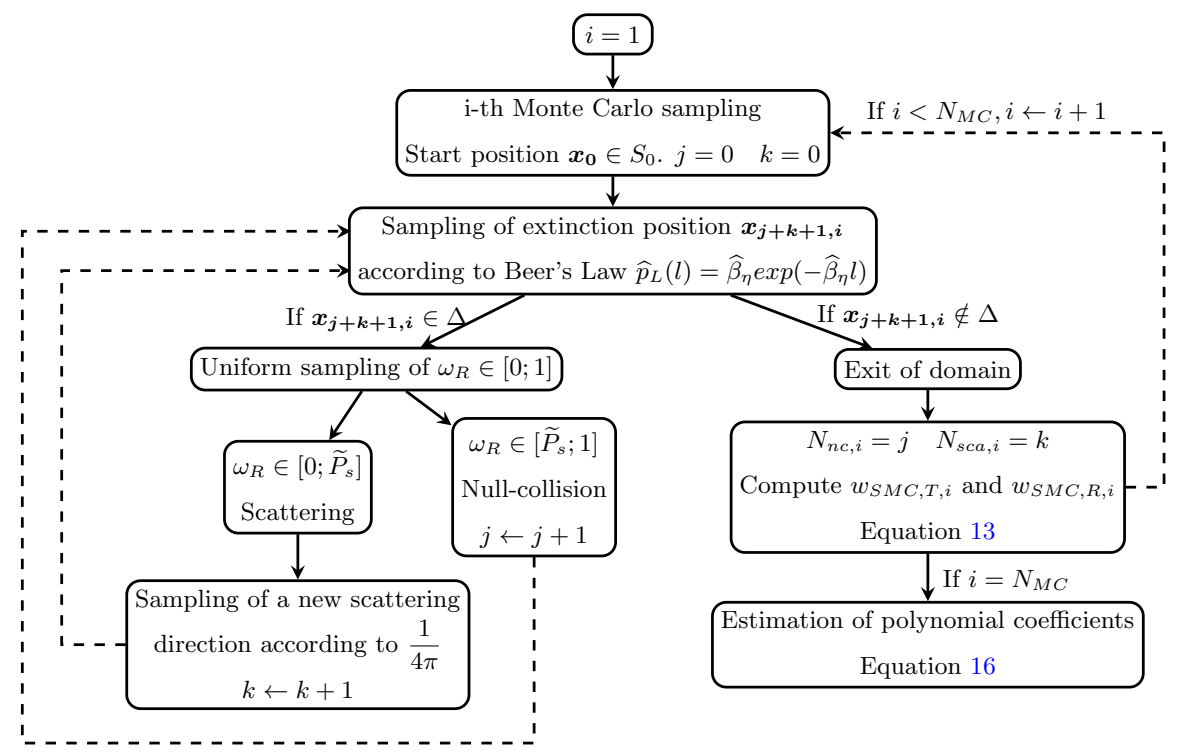

Figure 5: Symbolic Monte Carlo algorithm for the estimation of directional-hemispherical transmittance and reflectance 


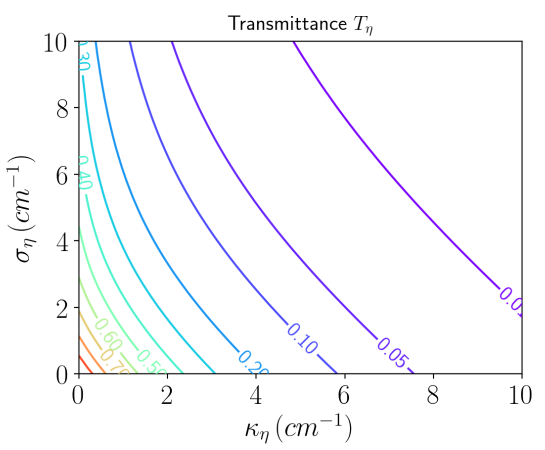

(a)

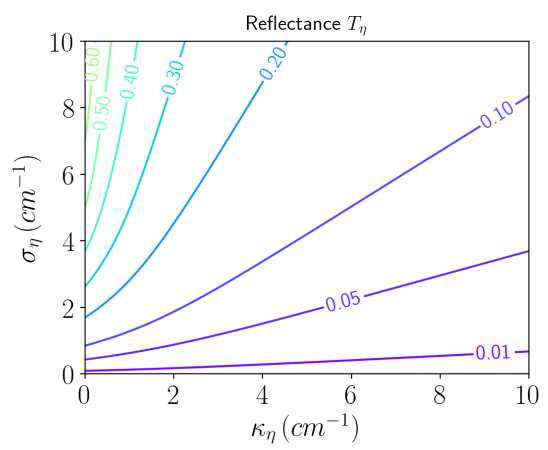

(b)

Figure 6: Isovalues of directional-hemispherical (a) transmittance $T_{\eta}$ and (b) reflectance $R_{\eta}$ computed with SMC as a function of absorption and scattering coefficients 


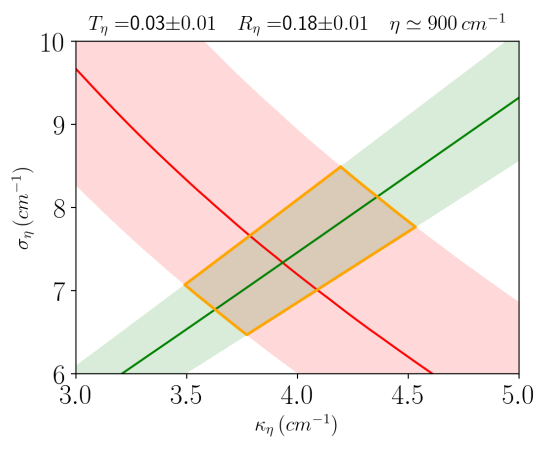

(a)

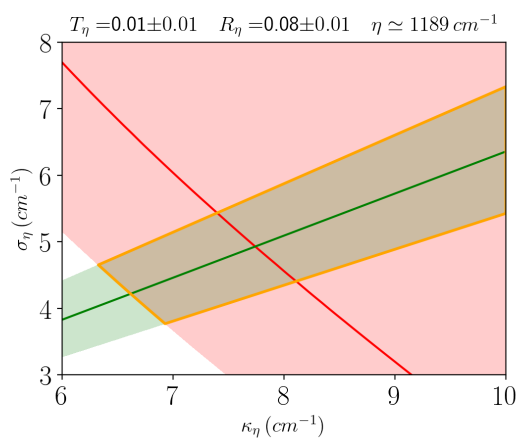

(b)

Figure 7: Region of possible solution $\kappa_{\eta}$ and $\sigma_{\eta}$ at (a) $\eta=900 \mathrm{~cm}^{-1}$ and (b) $\eta=1189 \mathrm{~cm}^{-1}$, when experimental and numerical uncertainties are included in the analysis. 


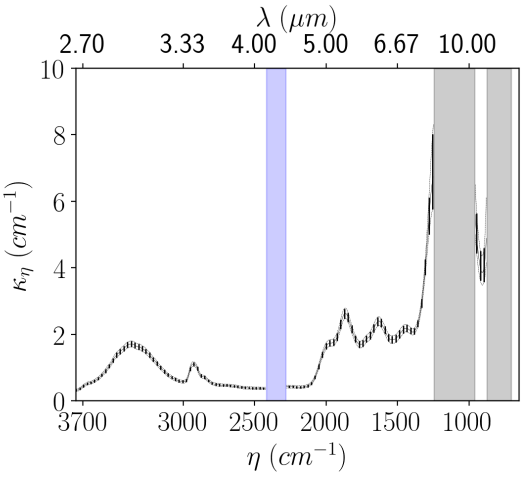

(a)

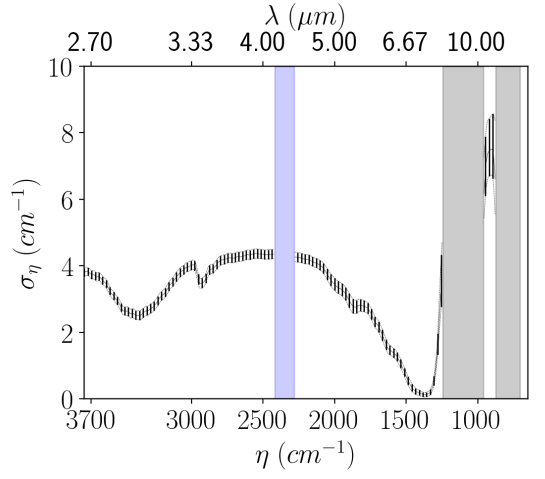

(b)

Figure 8: Identified (a) absorption and (b) scattering coefficients using SMC polynomials and taking into account measurements uncertainties. Colored band (in blue) in spectral range $[2222 ; 2398] \mathrm{cm}^{-1}([4.17 ; 4.5] \mu \mathrm{m})$ represents absorption peak by $\mathrm{CO}_{2}$ absorption. Grey bands display spectral ranges where the identification is not feasible. 


\section{Appendix A. Consequences of the arbitrary choice of $\widetilde{P_{s}}$ on SMC standard-deviation}

With SMC, standard-deviation can be expressed as a polynomial of absorption and scattering coefficients [12]:

$$
\begin{aligned}
\mathrm{S}_{\mathrm{SMC}}\left(T_{\eta}\left(\kappa_{\eta}, \sigma_{\eta}\right)\right)= & {\left[\frac{1}{N_{M C}-1} \sum_{j=0}^{\infty} \sum_{k=0}^{\infty} \sum_{j^{\prime}=0}^{\infty} \sum_{k^{\prime}=0}^{\infty}\left(\overline{a_{j k} a_{j^{\prime} k^{\prime}}}-\overline{a_{j k}} \overline{a_{j^{\prime} k^{\prime}}}\right)\right.} \\
& \left.\times\left(\frac{\widehat{\beta}_{\eta}-\kappa_{\eta}-\sigma_{\eta}}{\widehat{\beta}_{\eta}\left(1-\widetilde{P}_{s}\right)}\right)^{j+j^{\prime}}\left(\frac{\sigma_{\eta}}{\widehat{\beta}_{\eta} \widetilde{P}_{s}}\right)^{k+k^{\prime}}\right]^{1 / 2}
\end{aligned}
$$

where:

$$
\overline{a_{j k} a_{j^{\prime} k^{\prime}}}=\frac{1}{N_{M C}} \sum_{i=1}^{N_{M C}}\left(a_{j k, i} a_{j^{\prime} k^{\prime}, i}\right)
$$

and $a_{j k, i}$ are given by Eq 14 and $\overline{a_{j k}}$ by Eq 16 .

Under pure statistical considerations, such functional expressions of standarddeviations present a valuable advantage: the evolution of the uncertainty with the considered parameters is explicit, and may provide relevant information on the definition of the arbitrary probability $\widetilde{P_{s}}$.

In the algorithm described in subsection 3.3, absorption and scattering coefficients are unknown and arbitrary probabilities of scattering $\widetilde{P_{s}}$ and null-collision $\widetilde{P_{n}}=1-\widetilde{P_{s}}$ are required. The introduction of an arbitrary probability does not create any bias as an infinity of Monte Carlo samples would lead to an exact solution, but may have significant influence on the statistical uncertainties, and therefore the convergence speed can be altered. However, in order to ensure a small standard deviation, arbitrary scattering probability should be consistent with the statistics of the considered physics [12] and should be close to the ratio of the number of scattering events over the whole number of collisions given by equation 5 .

The influence of this choice is illustrated in figure A.9. Three SMC calculations have been carried out with three different $\widetilde{P_{s}}\left(\widetilde{P_{s}}=0.2, \widetilde{P_{s}}=0.5\right.$ and $\left.\widetilde{P_{s}}=0.8\right)$ with $N_{M C}=10^{6}$. The three estimated SMC relative standarddeviation are compared over the spectrum. For $\widetilde{P_{s}}=0.5$, used in this work, 
relative standard-deviation has a maximum value of $2.1 \%$ for transmittance and

$3982.6 \%$ for reflectance which is satisfactory for the identification of absorption and

399 scattering coefficient. If $\widetilde{P_{s}}$ is taken equal to 0.2 , maximum relative standard-

400 deviation observed over the spectrum is $0.6 \%$ for transmittance and $0.3 \%$ for

401 reflectance. However, if $\widetilde{P_{s}}=0.8$, maximum relative standard-deviation ob-

402 served over the spectrum is close to $40 \%$ for transmittance and reflectance. For

${ }_{403}$ the studied sample, the best choice of $\widetilde{P_{s}}$ would have been 0.2 since it is the

${ }_{404}$ closest to the real value of $P_{s, \eta}=\frac{\sigma_{\eta}}{\widehat{\beta}-\kappa_{\eta}}$ in the most part of the spectrum. 


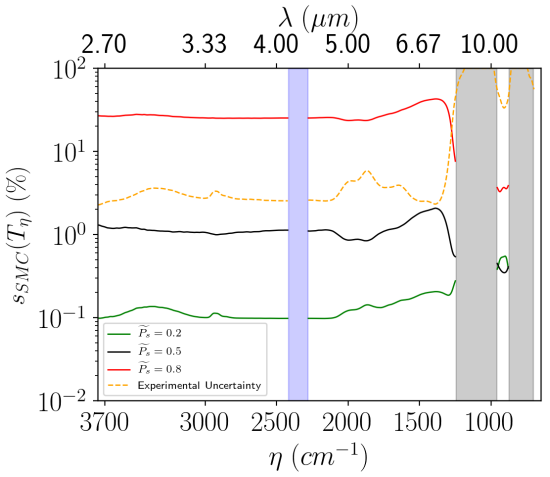

(a)

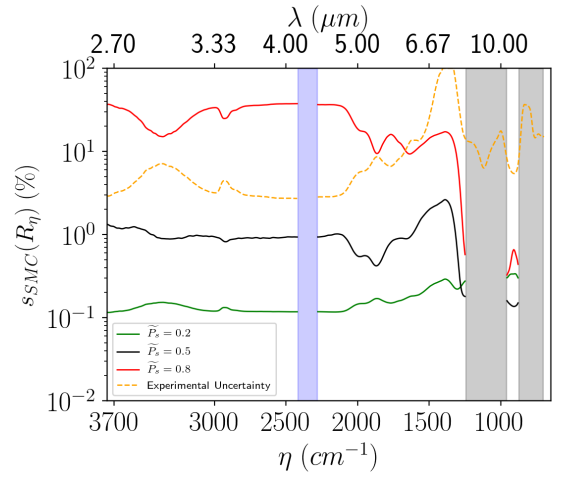

(b)

Figure A.9: Relative standard-deviation estimated using SMC for (a) transmittance and (b) reflectance, for different values of $\widetilde{P_{s}}$. 


\section{References}

[1] D. Baillis, M. Arduini-Schuster, J. Sacadura, Identification of spectral radiative properties of polyurethane foam from hemispherical and bidirectional transmittance and reflectance measurements, Journal of Quantitative Spectroscopy and Radiative Transfer 73 (2002) 297 - 306. doi: https://doi.org/10.1016/S0022-4073(01)00199-6.

[2] J. Sacadura, D. Baillis, Experimental characterization of thermal radiation properties of dispersed media, International Journal of Thermal Sciences 41 (2001) 699 - 707. doi:https://doi.org/10.1016/S1290-0729(02) 01365-0.

[3] T. Ren, M. F. Modest, A. Fateev, S. Clausen, An inverse radiation model for optical determination of temperature and species concentration: Development and validation, Journal of Quantitative Spectroscopy and Radiative Transfer 151 (2015) 198 - 209. doi:https://doi.org/10.1016/j.jqsrt. 2014.10 .005 .

[4] T. Tarvainen, V. Kolehmainen, S. R. Arridge, J. P. Kaipio, Image reconstruction in diffuse optical tomography using the coupled radiative transportdiffusion model, Journal of Quantitative Spectroscopy and Radiative Transfer 112 (16) (2011) 2600 - 2608. doi:https://doi.org/10.1016/j. jqsrt.2011.07.008.

[5] A. Addoum, O. Farges, F. Asllanaj, Optical properties reconstruction using the adjoint method based on the radiative transfer equation, Journal of Quantitative Spectroscopy and Radiative Transfer 204 (2018) 179 - 189. doi:https://doi.org/10.1016/j.jqsrt.2017.09.015.

[6] A. Tilioua, L. Libessart, G. Jeandel, S. Lassue, Determination of radiative properties of polyester batting insulation material from hemispherical transmittance and reflectance measurements, Applied Thermal Engineering 105 (2016) 594 - 604. doi:https://doi.org/10.1016/j. applthermaleng.2016.03.050. 
[7] P. Boulet, J. Grardin, Z. Acem, G. Parent, A. Collin, Y. Pizzo, B. Porterie, Optical and radiative properties of clear PMMA samples exposed to a radiant heat flux, International Journal of Thermal Sciences 82 (2014) 1-8. doi:https://doi.org/10.1016/j.ijthermalsci.2014.03.013.

[8] A. Milandri, F. Asllanaj, G. Jeandel, Determination of radiative properties of fibrous media by an inverse methodcomparison with the Mie theory, Journal of Quantitative Spectroscopy and Radiative Transfer 74 (2002) 637 - 653. doi:https://doi.org/10.1016/S0022-4073(01) 00276-X.

[9] W. Dunn, J. Shultis, Exploring Monte Carlo Methods, Elsevier Science \& Technology, 2011.

[10] W. Dunn, Inverse Monte Carlo solutions for radiative transfer in inhomogeneous media, Journal of Quantitative Spectroscopy and Radiative Transfer 29 (1983) 19 - 26. doi:https://doi.org/10.1016/0022-4073(83) 90141-3.

[11] S. Subramaniam, P. Menguc, Solution of the inverse radiation problem for inhomogeneous and anisotropically scattering media using a Monte Carlo technique, International Journal of Heat and Mass Transfer 34 (1991) 253 - 266. doi:https://doi.org/10.1016/0017-9310(91)90192-H.

[12] M. Galtier, M. Roger, F. André, A. Delmas, A symbolic approach for the identification of radiative properties, Journal of Quantitative Spectroscopy and Radiative Transfer 196 (2017) 130 - 141. doi:https://doi.org/10. $1016 / j \cdot j$ gsrt. 2017.03.026.

[13] M. Galtier, S. Blanco, C. Caliot, C. Coustet, J. Dauchet, M. E. Hafi, V. Eymet, R. Fournier, J. Gautrais, A. Khuong, B. Piaud, G. Terre, Integral formulation of null-collision Monte Carlo algorithms, Journal of Quantitative Spectroscopy and Radiative Transfer 125 (2013) 57 - 68. doi:https://doi.org/10.1016/j.jqsrt.2013.04.001. 
[14] P. Honnerová, J. Martan, Z. Veselý, M. Honner, Method for emissivity measurement of semitransparent coatings at ambient temperature, Scientific Reports 7. doi:https://doi.org/10.1038/s41598-017-01574-x.

[15] V. Eymet, D. Poitou, M. Galtier, M. E. Hafi, G. Terre, R. Fournier, Nullcollision meshless Monte Carlo : Application to the validation of fast radiative transfer solvers embedded in combustion simulators, Journal of Quantitative Spectroscopy and Radiative Transfer 129 (2013) 145 - 157. doi:https://doi.org/10.1016/j.jqsrt.2013.06.004.

[16] B. Sumlin, W. Heinson, R. Chakrabarty, Retrieving the aerosol complex refractive index using pymiescatt: A Mie computational package with visualization capabilities, Journal of Quantitative Spectroscopy and Radiative Transfer 205 (2018) 127 - 134. doi:https://doi.org/10.1016/j.jqsrt. 2017.10 .012 .

[17] M. Roger, Y. Maanane, M. Galtier, F. André, A. Delmas, Symbolic Monte Carlo method based on orthogonal polynomial series: application to phase function, 2019. doi:10.1615/RAD-19.360.

[18] S. Le Foll, A. Delmas, F. André, Identification of radiative properties for heterogeneous materials at high temperature, International Journal of Thermal Sciences 120 (2017) 314-320. doi:10.1016/j.ijthermalsci. 2017.06 .019 .

[19] M. Roger, M. Galtier, F. André, A. Delmas, Symbolic Monte Carlo methods: an analysis tool for the experimental identification of radiative properties at high temperature, Eurotherm Seminar 110 - Computational Thermal Radiation in Participating Media VI at Cascais (Portugal), 2018.

[20] N. Diascorn, Elaboration and characterization of silica and polyurethane based thermal superinsulating hybrid aerogels, Phd thesis, Ecole Nationale Supérieure des Mines de Paris (Dec 2014).

URL https://pastel.archives-ouvertes.fr/tel-01151554 